\title{
Structurally Diverse Covalent Triazine-Based Framework Materials for Photocatalytic Hydrogen Evolution from Water
}

\author{
Christian B. Meier, ${ }^{\dagger}$ Rob Clowes, ${ }^{\dagger}$ Enrico Berardo, ${ }^{\S}$ Kim E. Jelfs, ${ }^{\S}{ }^{\circledR}$ Martijn A. Zwijnenburg, ${ }^{*},+(1)$ \\ Reiner Sebastian Sprick, ${ }^{*}+$ a \\ †Department of Chemistry and Materials Innovation Factory, University of Liverpool, 51 Oxford Street, Liverpool L7 3NY, U.K. \\ ${ }^{\star}$ Department of Chemistry, University College London, 20 Gordon Street, London WC1H 0AJ, U.K. \\ ${ }^{\S}$ Department of Chemistry, Molecular Sciences Research Hub, Imperial College London, White City Campus, Wood Lane, London \\ W12 0BZ, U.K.
}

\section{Supporting Information}

ABSTRACT: A structurally diverse family of 39 covalent triazine-based framework materials (CTFs) are synthesized by Suzuki-Miyaura polycondensation and tested as hydrogen evolution photocatalysts using a high-throughput workflow. The two best-performing CTFs are based on benzonitrile and dibenzo[b,d]thiophene sulfone linkers, respectively, with catalytic activities that are among the highest for this material class. The activities of the different CTFs are rationalized in terms of four variables: the predicted electron affinity, the predicted ionization potential, the optical gap, and the dispersibility of the CTFs particles in solution, as measured by optical transmittance. The electron affinity and dispersibility in solution are found to be the best predictors of photocatalytic hydrogen evolution activity.
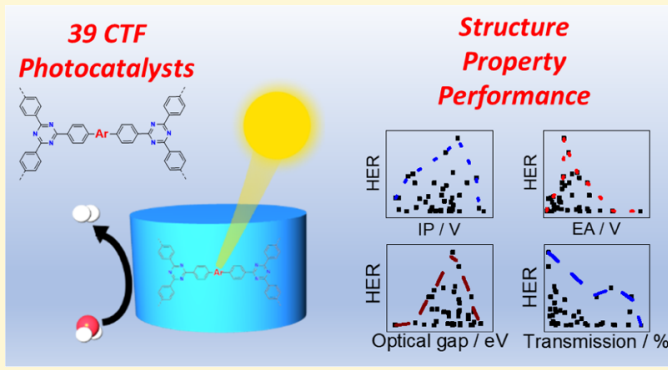

\section{INTRODUCTION}

Hydrogen is essential in many industrial processes, such as the hydrogenation of unsaturated fats, ${ }^{1}$ production of stainless steel $(\mathrm{ss})^{2}$ and ammonia, ${ }^{3}$ and crude oil purification. ${ }^{4}$ Hydrogen also has potential as a clean fuel for fuel cells that does not result in the emission of greenhouse gases at the point of use. ${ }^{5}$ Currently, hydrogen is made mostly by steam reforming of methane, which releases carbon dioxide directly and indirectly as a result of the high temperatures and pressures required for this process. ${ }^{6}$

Photocatalytic water splitting has been studied extensively as a green method for the production of hydrogen, releasing oxygen as the only side product. Light-driven water splitting was first demonstrated in 1972 for a photoelectrochemical cell with a $\mathrm{TiO}_{2}$ anode and platinum cathode. ${ }^{7}$ After this first report, many inorganic photocatalysts have been studied, also in suspension systems that do not require an applied bias. ${ }^{8-10}$ Conjugated polymer photocatalysts were studied as early as $1985,{ }^{19-14}$ but it took the first report on carbon nitride in $2009^{15}$ to trigger real interest in this field. Some limitations arise from the synthesis temperatures needed for carbon nitrides, which are typically above $300{ }^{\circ} \mathrm{C}$, and this can result in a lack of synthetic control. A large number of studies reported improved hydrogen production rates under sacrificial conditions by varying the synthesis conditions and starting materials, ${ }^{16-21}$ by introducing doping, ${ }^{22,23}$ by controlling end groups, ${ }^{24-26}$ or by using heterojunctions ${ }^{27}$ or donor/acceptortype systems. ${ }^{28}$ Conjugated organic polymers are typically made under much milder conditions ${ }^{29}$ than carbon nitrides and offer potential advantages in terms of synthetic control via the incorporation of different building blocks ${ }^{30-32}$ and by monomer sequence control. ${ }^{33}$ Conjugated polymers can be tailored in terms of their optical properties, as well as their driving force for proton reduction and water/sacrificial electron donor oxidation, respectively. ${ }^{33,34} \mathrm{~A}$ number of conjugated organic materials have now been studied for sacrificial hydrogen and/or oxygen production from water using light, including oligomers, ${ }^{35-37}$ linear polymers, ${ }^{34,38-45}$ covalent organic frameworks, ${ }^{46-54}$ and networks. ${ }^{42,55-62}$

Covalent triazine-based frameworks (CTFs) are a subclass of conjugated microporous polymers that have been reported to be photocatalytically active for hydrogen and oxygen evolution from water. ${ }^{30,63,64}$ The most common reactions used to prepare CTFs are the acid-catalyzed trimerization of aromatic nitriles, ${ }^{57,65-68}$ sometimes combined with sulfur and phosphorous doping, ${ }^{69,70}$ and high-temperature ionothermal synthesis. ${ }^{71-73} \mathrm{~A}$ two-step route was also reported whereby acidcatalyzed trimerizations were used to make CTFs that were then further condensed using thermal methods, ${ }^{74,75}$ or doped using ammonium halides. ${ }^{76}$ We previously used acid-catalyzed trimerization to prepare a series of CTFs with an increasing linker length between the triazine vertices (phenylene to

Received: July 16, 2019

Revised: September 27, 2019

Published: September 27, 2019 
(a)

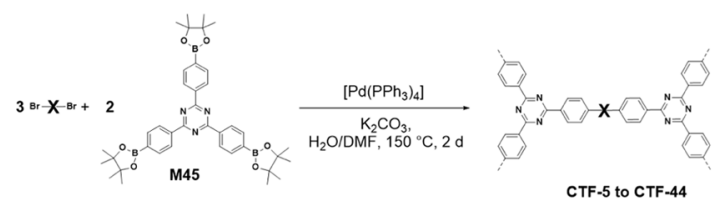
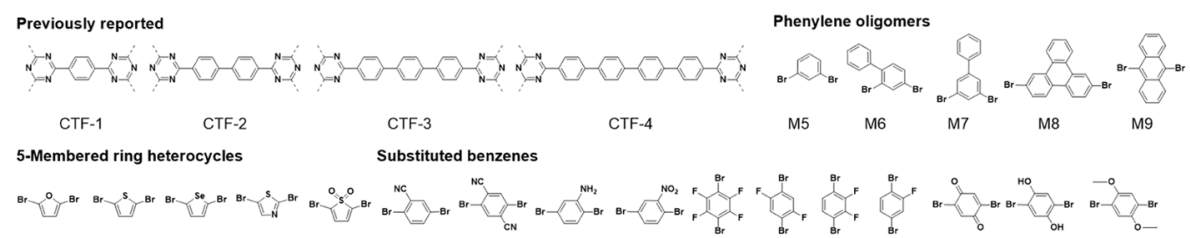

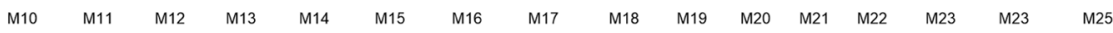
Bipyridyls

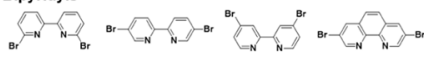

$\begin{array}{llll}\text { M26 } & \text { M27 } & \text { M28 }\end{array}$

$N$-Heterocycles

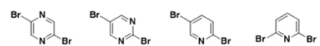

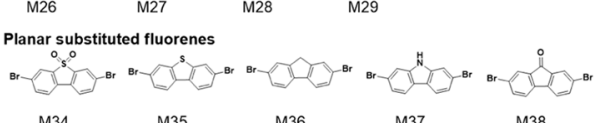

M30 M31 M32 M33

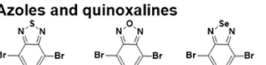

M39 M40 M4

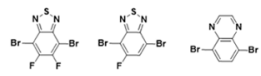

(b)
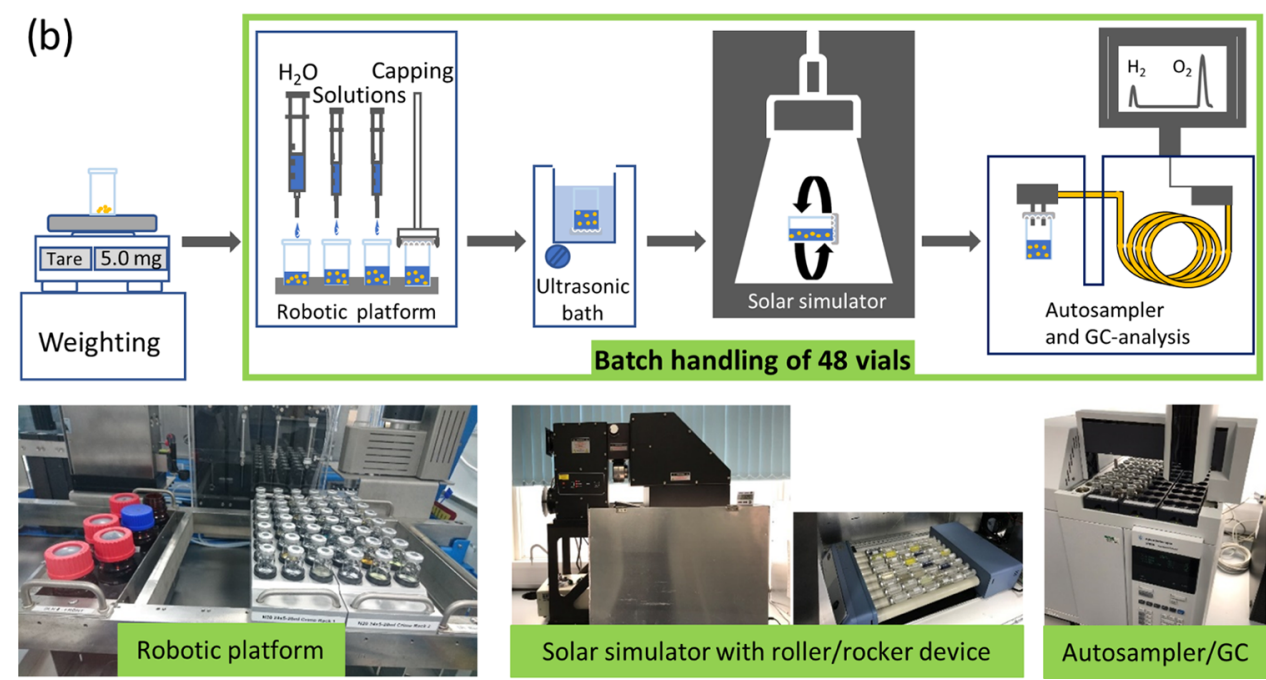

Figure 1. (a) Synthesis of the CTF photocatalyst library, showing the linkers used. (b) High-throughput (HT) workflow for hydrogen production of suspension photocatalysts (top row) and photographs of equipment used in the workflow (bottom row).

quarterphenylene; Figure 1, CTF-1-4). In that series, the highest hydrogen evolution activity was observed for the acidcatalyzed CTF-2, which is based on a biphenylene linker. ${ }^{57}$

Alkyne-linked CTFs have also recently been made using Sonogashira-Hagihara polycondensation linking tris(4-ethynylphenyl)-1,3,5-triazine with a range of nitrogen- and sulfurcontaining compounds. ${ }^{77}$

To date, studies on CTFs have shown rather limited structural diversity either because of the availability of monomers, ${ }^{57,78}$ and/or the harsh synthesis conditions employed; for example, in ionothermal synthesis, partial carbonization might limit the materials' photocatalytic activity, ${ }^{65,74}$ while acid-catalyzed cyclotrimerization is incompatible with functional groups that are acid sensitive. In this study, we present 39 new CTFs prepared by $\operatorname{Pd}(0)$-catalyzed Suzuki-Miyaura polycondensation. We study these in detail for their photocatalytic hydrogen production activity (Figure 2 ). As such, this single study covers a significantly more diverse set of CTF materials than the 18 structurally defined photocatalytically active CTFs published so far in the combined literature. $^{30,57,60,67,73,79-81}$

\section{EXPERIMENTAL SECTION}

General Procedure for the Pd(0)-Catalyzed Suzuki-Miyaura Polycondensation. A flask was charged with the dibromo monomer, 2,4,6-tris[4-(4,4,5,5-tetramethyl-1,3,2-dioxaborolan-2-yl)phenyl]-1,3,5-triazine, $\mathrm{N}, \mathrm{N}$-dimethylformamide, and an aqueous solution of $\mathrm{K}_{2} \mathrm{CO}_{3}(2.0 \mathrm{M})$ and degassed by bubbling with $\mathrm{N}_{2}$ for $30 \mathrm{~min} .\left[\mathrm{Pd}\left(\mathrm{PPh}_{3}\right)_{4}\right](1.2 \mathrm{~mol} \%)$ was added and the solution was degassed for $10 \mathrm{~min}$ further. The reaction mixture was then heated to $150{ }^{\circ} \mathrm{C}$ for 2 days, cooled to room temperature, and poured into water. The precipitate was collected by filtration and washed with water and methanol. Further purification was carried out by Soxhlet extraction with cyclopentyl methyl ether. The product was then recovered from the Soxhlet thimble and dried under reduced pressure at $75{ }^{\circ} \mathrm{C}$. See the Supporting Information for results of polymer characterization.

Kinetic Hydrogen Evolution Experiments. A quartz flask was charged with the polymer powder $(25 \mathrm{mg})$, water $(8.3 \mathrm{~mL})$, triethylamine (TEA, $8.3 \mathrm{~mL}$ ), methanol $\left(8.3 \mathrm{~mL}\right.$ ), and $\mathrm{H}_{2} \mathrm{PtCl}_{6}$ solution $\left(0.02 \mathrm{~mL}, 8 \mathrm{wt} \%\right.$ in $\left.\mathrm{H}_{2} \mathrm{O}\right)$ and sealed with a septum. The resulting suspension was ultrasonicated for $10 \mathrm{~min}$ until the photocatalyst was well-dispersed before degassing by $\mathrm{N}_{2}$ bubbling for $30 \mathrm{~min}$. The reaction mixture was side illuminated with a $300 \mathrm{~W}$ Newport Xe light source (model: 6258, ozone free) equipped with a $\lambda$ 

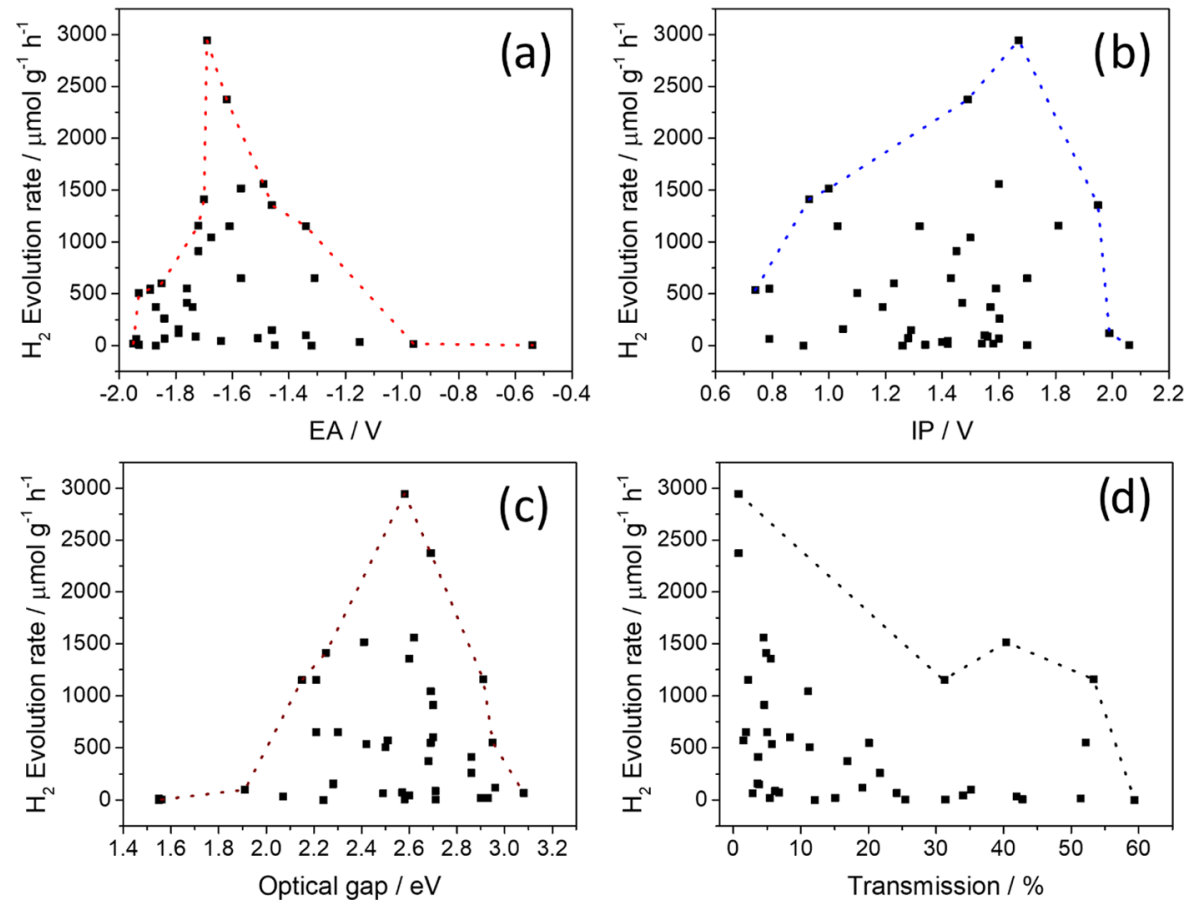

Figure 2. (a) Hydrogen evolution rate plotted as a function of the predicted electron affinity; (b) the predicted ionization potential; (c) the measured optical gap; and $(\mathrm{d})$ the measured optical transmission of the sample dispersed in the water/TEA/MeOH mixture. All hydrogen evolution rates were determined as linear fits of hydrogen produced over $5 \mathrm{~h}$ for $25 \mathrm{mg}$ photocatalyst in water/TEA/MeOH and $3 \mathrm{wt} \%$ platinum co-catalyst under visible light $(\lambda>420 \mathrm{~nm}, 300 \mathrm{~W}$ Xe light source).

$>420 \mathrm{~nm}$ filter for $5 \mathrm{~h}$ unless stated otherwise. The light source was cooled and IR light cut out by water circulating through a metal jacket with quartz windows. For the illumination under solar light, an Oriel LSH-7320 ABA LED solar simulator with an output of 1.0 sun was used (classification ICE 60904-9 2007: spectral match A, uniformity classification B, temporal stability A). Gas samples were taken with a gas-tight syringe and run on a Bruker 450-GC gas chromatograph equipped with a molecular sieve $13 \times 60-80$ mesh $1.5 \mathrm{~m} \times 1 / 8 \mathrm{in} . \times$ $2 \mathrm{~mm}$ ss column at $50{ }^{\circ} \mathrm{C}$ with an argon flow of $40.0 \mathrm{~mL} \mathrm{~min}{ }^{-1}$. Hydrogen was detected with a thermal conductivity detector referencing against standard gas with a known concentration of hydrogen. Hydrogen dissolved in the reaction mixture was not measured, and the pressure increase generated by the evolved hydrogen was neglected in the calculations. The rates were determined from a linear regression fit and the error is given as the standard deviation from the linear fit. No hydrogen evolution was observed for mixtures of water/triethylamine $/ \mathrm{H}_{2} \mathrm{PtCl}_{6}$ under $\lambda>420$ $\mathrm{nm}$ illumination or under the solar simulator illumination in the absence of a photocatalyst. No hydrogen was observed for L-ascorbic acid solution under $\lambda>420,>350$, and $>295 \mathrm{~nm}$ illumination $(300 \mathrm{~W}$ Xe light source) or solar-simulated light (ABA, 400-1100 nm) in the absence of a photocatalyst. Aqueous $\mathrm{Na}_{2} \mathrm{~S}$ solution showed a limited hydrogen production rate of $0.15 \mu \mathrm{mol} \mathrm{h}{ }^{-1}$ under $\lambda>295 \mathrm{~nm}$ illumination ( $300 \mathrm{~W}$ Xe light source). For longer-term stability experiments, the photocatalysis was carried out for $5 \mathrm{~h}$ and the polymer was recovered through centrifugation and after decantation of the liquids, water, methanol, and triethylamine (TEA) mixtures were added for the next run. After $100 \mathrm{~h}$ of combined photocatalysis, the photocatalyst was isolated by decantation of the aqueous phase, washed with water and THF three times each, and dried overnight at $120{ }^{\circ} \mathrm{C}$ before analysis.

High-Throughput Hydrogen Evolution Experiments. The Agilent Technologies vials (crimp top, headspace, clear with graduation marks and write-on spot, flat bottom, $10 \mathrm{~mL}, 23 \times 46$ $\mathrm{mm}$ ) were charged with $5.0 \pm 0.1 \mathrm{mg}$ of polymer powders and transferred to the Chemspeed Accelerator SWING for liquid transfer. Degassed jars with triethylamine, methanol, and a stock solution of $\mathrm{H}_{2} \mathrm{PtCl}_{6}$ were loaded into the automated liquid handling platform.
The system was then closed and purged for $4 \mathrm{~h}$ with nitrogen. The automated liquid handling platform then dispenses the liquids as specified, which was unless stated otherwise degassed water $(1.7 \mathrm{~mL})$ or aqueous $\mathrm{H}_{2} \mathrm{PtCl}_{6}$ solution $\left(1.7 \mathrm{~mL}, 0.24\right.$ wt $\%$ in $\mathrm{H}_{2} \mathrm{O}$ ) triethylamine $(1.7 \mathrm{~mL})$, methanol $(1.7 \mathrm{~mL})$. The vials were then capped using the capper/crimper tool under inert conditions. Once capped, the samples are taken out, shaken for $5 \mathrm{~s}$, and transferred to an ultrasonic bath to disperse the photocatalysts. An Oriel Solar Simulator 94123A with an output of 1.0 sun was then used to illuminate the vials on a Stuart roller bar SRT9 for the time specified (classification IEC 60904-9 2007 spectral match A, uniformity classification A, temporal stability A, $1600 \mathrm{~W}$ xenon light source, 12 $\times 12$ in. $^{2}$ output beam, air mass 1.5 G filter, $350-1000 \mathrm{~nm}$ ). After photocatalysis, the gaseous products of the samples are measured on an Agilent Technologies $7890 \mathrm{~B}$ gas chromatograph connected to an Agilent Technologies 7697A headspace sampler. No hydrogen evolution was observed for mixtures of water/triethylamine/methanol or water/triethylamine/methanol $/ \mathrm{H}_{2} \mathrm{PtCl}_{6}$ under solar irradiation (1.0 sun) in the absence of a photocatalyst. No hydrogen production was observed in the absence of a photocatalyst.

(Time-Dependent) Density Functional Theory [(TD-)DFT] Calculations. The CTFs were modeled for the purpose of computationally screening their properties as triazine-linker-triazine (TLT) cluster models. The relevant three-dimensional structures of these TLT CTF models were generated using a multistep process starting from the SMILES codes for the linkers using the supramolecular toolkit (stk). ${ }^{82,83}$ stk is a Python library for the assembly, structure generation, and property calculation of supramolecules (using functionality from $\mathrm{RDKit}^{84}$ and Schrödinger PLC's Macromodel software) and allowed us to automate the assembly and conformer search process. The as-assembled TLT cluster models were energy minimized using the OPLS2005 ${ }^{85,86}$ force field. After the relaxation step, a high-temperature OPLS2005 molecular dynamics run with regular quenches $(700 \mathrm{~K}, 50 \mathrm{ps}$ of simulation time, and 1000 quenches) was employed to find low-energy conformers for each cluster model. The lowest-energy conformers were finally reoptimized using density functional theory (DFT), using the B $3 \mathrm{LYP}^{87,88}$ density functional and the $\mathrm{DZP}^{89}$ basis set. 
The thermodynamic ability of the various CTFs, as modeled by TLT cluster models, to reduce protons and oxidize water and/or triethylamine was analyzed in the terms of the potentials associated with free electrons [electron affinity (EA)] and holes [ionization potential (IP)], as well as the exciton (IP* and $\mathrm{EA}^{*}$ ), relative to those of the different solution half reactions. These potentials are calculated using our standard $\triangle$ SCF approach ${ }^{90,91}$ based on DFT/timedependent DFT (TD-DFT) calculations on polymer cluster models and the use of the $\operatorname{COSMO}^{92}$ continuum solvation model $\left(\varepsilon_{\mathrm{r}} 80.1\right.$, water) to approximate the dielectric environment of the polymers in the presence of water. As in our previous work ${ }^{33,34,38,43,57,62,90,91}$ and as in the CTF model generation stage, the potential calculations use the B3LYP density functional and the DZP basis set, a combination that we previously found to result in the prediction of accurate potentials for polymeric solids when compared to experimental photoelectron spectroscopy data. ${ }^{91}$ Potentials of solution halfreactions are taken from previous work. ${ }^{33}$

The absorption spectra of the CTFs are approximated by the vertical excitation spectra of the TLT cluster models, as calculated by TD-B3LYP/DZP. Similarly, the optical gap, the energy of light above (and the wavelength below) which the CTF starts absorbing light, is approximated by the energy of the lowest vertical singlet excitation.

\section{RESULTS AND DISCUSSION}

A structurally diverse library of new CTFs was synthesized by using a trisphenyl-1,3,5-triazine building block as the node (M45) connected by various linkers (M5-44). The resulting CTFs are referred to as CTF- $x x$ (whereby $x x$ is the number of the dibromo monomers used in the synthesis, Mxx). The linkers cover a wide range of different aromatic units, which can be divided into the following subsets: (i) phenylene oligomers; (ii) five-membered aromatic heterocycles; (iii) substituted phenylenes; (iv) bipyridyls; (v) nitrogen heterocycles; (vi) planar heterofluorenes; and (vii) azoles and quinoxalines.

All polymers were prepared via $\operatorname{Pd}(0)$-catalyzed SuzukiMiyaura polycondensation of the corresponding dibromo monomer (M5-44) and 2,4,6-tris[4-(4,4,5,5-tetramethyl1,3,2-dioxaborolan-2-yl)-phenyl]-1,3,5-triazine (M45, Figure 1a)..$^{38,57,62}$ All of the materials were synthesized under the same conditions and without further optimization for any of the monomers. As a result, the polymer yields varied. In all, 22 polymers were obtained in isolated yields exceeding $90 \%$, and 16 polymers were obtained in isolated yields ranging from 37 to $87 \%$. Lower yields were observed for CTF-23 (2,5-linked 1,4-benzoquinone, $21 \%)$ and CTF-24 (2,5-linked benzene-1,4diol, $11 \%)$.

The polymers were characterized by Fourier transform infrared (FT-IR) spectroscopy (Figures S-1-S-7), thermogravimetric analysis (TGA) under air (Figures S-8-S-14), and nitrogen sorption at $77 \mathrm{~K}$ (Table S-1). The apparent Brunauer-Emmett-Teller (BET) surface areas $\left(\mathrm{SA}_{\mathrm{BET}}\right)$ also varied across the library: 14 materials were essentially nonporous $\left(\mathrm{SA}_{\mathrm{BET}}<100 \mathrm{~m}^{2} \mathrm{~g}^{-1}\right), 14$ materials had moderate surface areas $\left(\mathrm{SA}_{\mathrm{BET}}=100-300 \mathrm{~m}^{2} \mathrm{~g}^{-1}\right), 9$ materials had higher surface areas $\left(\mathrm{SA}_{\mathrm{BET}}=300-500 \mathrm{~m}^{2} \mathrm{~g}^{-1}\right)$, and 5 materials had BET surface areas greater than $500 \mathrm{~m}^{2} \mathrm{~g}^{-1}$. CTF9 (9,10-linked anthracene) had the highest $\mathrm{SA}_{\mathrm{BET}}\left(708 \mathrm{~m}^{2}\right.$ $\left.\mathrm{g}^{-1}\right)$.

UV-vis absorption spectra were measured in the solid state (Figures S-15-S-18 and Table S-2) and the optical gaps of the polymers were calculated from the absorption onset. CTF-24 had the lowest optical gap (2,5-linked benzene-1,4-diol -1.52 $\mathrm{eV})$, while CTF-26 had the highest (6,6'-linked 2,2'-bipyridine $-3.08 \mathrm{eV}$ ). Most of the materials (30 polymers) had optical gaps in the region of $1.95-2.70 \mathrm{eV}$. As can be seen from Figure 3 , there is a good correlation between the measured optical gap

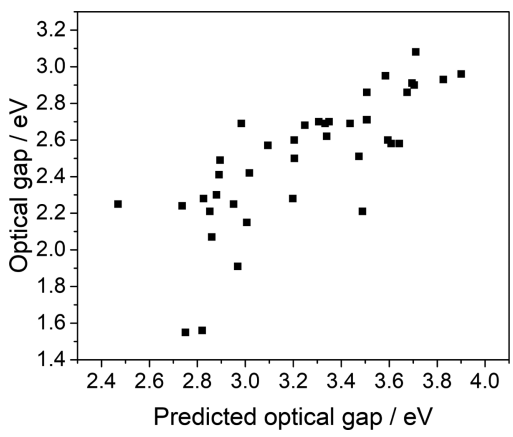

Figure 3. Predicted values of the optical gaps of the CTFs compared to measured values of the optical gaps.

values and those predicted by the TD-DFT calculations. All materials are weakly emissive and fluorescence decay measurements were carried out using time-correlated single photon counting (TCSPC) in the solid state (Figures S-49-S-70 and Table S-5). The CTFs had average weighted photoluminescence (PL) lifetimes ranging from 0.1 to $4.4 \mathrm{~ns}$ and $7.7 \mathrm{~ns}$ for the reference material CTF-2. Contact angles with water were measured for pressed pellets of the polymers and found to range from $55^{\circ}$ for 2,5-linked thiophene dioxide CTF-14 up to $106^{\circ}$ for 1,4-linked 2,5-dimethoxybenzene CTF-25 (Figures S$22-S-24$ and Table S-4); for 12 materials, a contact angle could not be measured because the water drop was absorbed by the porous polymer and swelling occurred.

To probe the dispersibility of the polymer particles in the reaction mixture, the transmission of light through a suspension of the photocatalysts in the water/TEA/methanol mixture was determined. The transmission values were found to range from low values of $0.8 \%$, indicating good dispersibility (i.e., almost opaque dispersions), to high values of up to 59.4\%, indicating poor dispersibility in water/TEA (triethylamine)/ methanol mixtures.

The photocatalytic activity for proton reduction of the materials was tested using a high-throughput workflow: the polymers $(5 \pm 0.5 \mathrm{mg})$ were dispersed in mixtures of water, TEA, and methanol (1/1/1 ratio). Platinum ( $3 \mathrm{wt} \%)$ was in situ photodeposited onto the photocatalyst ${ }^{57}$ acting as a cocatalyst, which has been shown previously to improve hydrogen evolution rates (HERs). ${ }^{57,67}$ TEA acts as a hole scavenger, ${ }^{35}$ and methanol is used to ensure miscibility between water and TEA. ${ }^{39,93}$ Samples were illuminated with a solar simulator for $2 \mathrm{~h}$ before measuring the amount of hydrogen produced using an automated gas chromatograph.

Two materials, CTF-15 (2,5-linked benzonitrile) and CTF34 (3,7-linked dibenzo[ $b, d]$ thiophene sulfone), were identified by this screen as the best-performing catalysts. The results of the screening measurements were compared to the traditional kinetic experiments for each photocatalyst over $5 \mathrm{~h}$ using a 300 $\mathrm{W}$ Xe light source equipped with a $\lambda>420 \mathrm{~nm}$ filter (Figures 4, S-73-S-94, and Table S-7). Again, the two most active photocatalysts were found to be CTF-15 $\left(2946 \mu \mathrm{mol} \mathrm{g}^{-1} \mathrm{~h}^{-1}\right)$ and CTF-34 $\left(2373 \mu \mathrm{mol} \mathrm{g}{ }^{-1} \mathrm{~h}^{-1}\right)$, which had significantly higher values than that of the previously reported CTF-3 Suzuki $\left(158 \mu \mathrm{mol} \mathrm{g} \mathrm{g}^{-1} \mathrm{~h}^{-1}\right)$ when tested under the same condition. Overall, there is a good correlation between the high-throughput screening measurements and the kinetic 


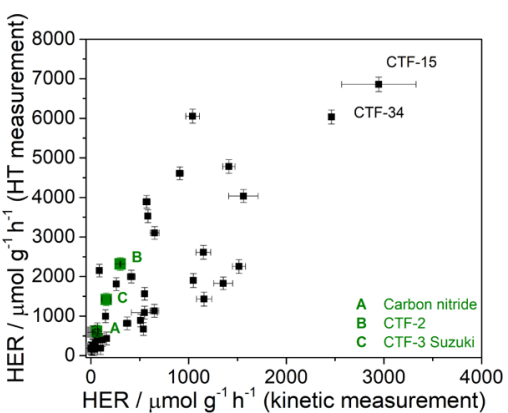

Figure 4. Comparison of the normalized rates of kinetic measurements ( $25 \mathrm{mg}$ photocatalyst) compared to high-throughput measurements ( $5 \mathrm{mg}$ photocatalyst) for each material. Conditions: water/ TEA/MeOH with 3 wt \% Pt co-catalyst.

measurements, especially when one considers the marked differences in the spectral output of the light sources for the two measurements, the different head spaces of the reaction vessels (much smaller in the high-throughput screen), and the different agitation methods (rolling/rocking in the screen versus stirring in the kinetic measurements)..$^{94}$

Following our previous work on linear conjugated polymer photocatalysts, ${ }^{34}$ we attempted to analyze the performance of the CTFs in terms of four properties: their electron affinity (EA), ionization potential (IP), and optical gap, as well as the optical transmittance of the reaction mixture after the CTF is dispersed in it. Figure $2 a-d$ shows the dependency of the measured hydrogen evolution rate on those four properties and dashed lines envelope the data points for all CTFs. The measured hydrogen evolution rate shows the clearest correlation with the predicted EA values (Figure 2a). EA, often approximated by (the negative of) the lowest unoccupied molecular orbital energy, governs the driving force for water reduction. None of the CTFs with EA values above $-1.4 \mathrm{~V}$ relative to the standard hydrogen electrode have high hydrogen evolution rates (here defined to have HER $>50 \%$ of the CTF with the highest activity in this study), while CTFs with even more positive EA values effectively produce no hydrogen. The observed HER is highest at around $-1.7 \mathrm{~V}$, but as the photocatalytic performance is not only controlled by the EA of the materials several CTFs with EA values in this range evolve only a small amount of hydrogen or show no activity (Figure 2a). Where EA governs the driving force for proton reduction, IP, often approximated by (the negative of) the highest occupied molecular orbital energy, determines the driving force for water oxidation or, as in this study, the oxidation of the hole scavenger (TEA). The measured hydrogen evolution rates lie in a broad envelope of increasing hydrogen evolution rates with increasing predicted IP. A maximum is observed at approximately $+1.6 \mathrm{~V}$, and then the measured hydrogen evolution rates decrease again for higher IP values (Figure $2 \mathrm{~b}$ ). In contrast to the case of EA, CTFs with high hydrogen evolution rates, as defined above, can be found across most of the IP range, and there are no IP regions in which exclusively CTFs occur that produce little or no hydrogen.

The apparent higher dependency of the measured hydrogen evolution rates of the materials on their predicted EA values (Figure 2a) when compared to the correlation of photocatalytic activity and predicted IP values of these materials (Figure $2 \mathrm{~b}$ ) can be explained when the effect of triethylamine on the $\mathrm{pH}$ of the photocatalysis mixtures is considered. The high $\mathrm{pH}$ of these mixtures of approximately 11.5 effects the potentials; hence, the proton reduction potential will lie at $-0.7 \mathrm{~V}$, which means that CTFs with EA values more positive than $\sim-1.0 \mathrm{~V}$ therefore are expected to have little or no driving force for proton reduction (Figure 2a). The overall oxidation of triethylamine to give diethylamine and acetaldehyde has a predicted potential to be at $-0.7 \mathrm{~V}$. Therefore, it is expected that the CTFs studied here all have significant driving force for scavenger oxidation. Even the one-hole oxidation step of triethylamine $(+0.7 \mathrm{~V})$ to the triethylamine radical $\left[\mathrm{N}\left(\mathrm{CH}_{2} \mathrm{CH}_{3}\right) \mathrm{CHCH}_{3}\right]$, which can be an effective thermodynamic obstacle for the overall oxidation reaction, is predicted to favor most CTFs considered.

Plots of measured hydrogen evolution rates versus predicted excited-state ionization potential (IP*) and electron affinity $\left(\mathrm{EA}^{*}\right)$, where the excited state (exciton) provides the electron or hole to drive the solution chemistry, look very similar to their EA and IP equivalents (Figure S-126). As a result, the correlation between the hydrogen evolution rate and the predicted $\mathrm{IP}^{*}$ values is also stronger than that between the measured and predicted EA* values. Exciton dissociation, or not, thus appears to make little difference for these materials in terms of the predicted potentials.

In the case of the (computed) optical gap (Figure 2c), we find that the CTFs with the highest activity have large optical gaps. Because, as discussed above, the predicted optical gap values and experimental absorption onsets are strongly correlated, the plot of the measured HER as a function of absorption onset looks similar (Figure S-117), and we made the same observation. The fact that the most active materials have large optical gaps is counterintuitive and would have been surprising had we not observed the same behavior for linear conjugated co-polymers. ${ }^{34}$ As for those linear polymers, plotting EA/IP as a functional of optical gap in this work (Figure S-125) shows that EA and optical gap are strongly correlated; that is, the CTFs with the largest optical gaps also have the most negative EA values. Hence, for both families of materials, the linear polymers and the CTFs, the hydrogen evolution rates, at least when using TEA, are limited more by the thermodynamic driving force for proton reduction than by light absorption.

The final property that we considered was the light transmittance of dispersions of the CTF in the photocatalysis mixture, i.e., TEA/MeOH/water (Figure 2d). Values range from $100 \%$ (indicating quick settling or "creaming" of the CTF) to $0 \%$ (indicating that the CTF is well-dispersed and scattered and/or absorbed light effectively). Light transmittance is a descriptor of the dispersibility of the CTF in the photocatalysis mixture, which is affected by interaction with water and scavenger, density, and size of the CTF particles. Hydrogen evolution rates in Figure $2 \mathrm{~d}$ are generally higher for CTFs when lowering the transmittance, and all CTFs that show high rates have a transmittance of less than $50 \%$, therefore showing a positive correlation between the dispersion of the CTF in the reaction mixture and the performance as a photocatalyst for proton reduction.

For completeness, we also considered a number of other properties beyond those considered in our previous work but found no correlation with the hydrogen evolution rate, other than that for the fitted fluorescence lifetimes measured via TCSPC. It appears that materials with shorter lifetimes have a higher photocatalytic activity (Figure S-119). However, as in the case for the optical gap, this correlation appears counterintuitive. As the TCSPC measurements are performed 
in the absence of scavenger, these short lifetimes are not due to quenching but rather a sign of inherently short-lived excited states, which should be a negative effect rather than a positive one. We suspect that this is a noncausal correlation with the hydrogen evolution rate, as for the optical gap, that arises because the fluorescence lifetimes are correlated with another material property.

The above analysis demonstrates that CTFs with the highest photocataytic activities occur for specific combinations of (material) properties. The word combinations in the above sentence is important, as there are clearly also CTFs with (near-)"ideal" EA, IP, optical gap, and/or transmittance values that yet only evolve a very small amount of hydrogen or are not active at all (see also Figures 2 and S-124). In line with our previous observations for linear conjugated polymers, each of these four properties appear to be a necessary but not sufficient condition for a CTF to be an active photocatalyst.

The high-throughput system was further used to study the best-performing material, CTF-15 (2,5-linked benzonitrile), with various different scavenger systems in combination with platinum as the co-catalyst (Figure S-97 and Table S-9). The screening shows that the highest performance was obtained in this case for water/TEA/MeOH mixtures $\left(1909 \mu \mathrm{mol} \mathrm{g}^{-1}\right.$ $\left.\mathrm{h}^{-1}\right)$, followed by 5 vol \% TEA in water $\left(1830 \mu \mathrm{mol} \mathrm{g}{ }^{-1} \mathrm{~h}^{-1}\right)$. Moderate hydrogen production was observed when L-ascorbic acid and sodium sulfide were used as scavengers (116 and 139 $\mu$ mol g ${ }^{-1} \mathrm{~h}^{-1}$, respectively) and low performance was observed for disodium ethylenediaminetetraacetate, L-cysteine, and thiophenol (101, 82, and $\left.77 \mu \mathrm{mol} \mathrm{g}{ }^{-1} \mathrm{~h}^{-1}\right)$. $\mathrm{FeCl}_{2}$, methanol, phenol, and hydroquinone did not act as a scavenger and no hydrogen production was observed under these conditions. Furthermore, CTF-15 and CTF-34 were compared to CTF-16 and CTF-3 Suzuki in a HT screening using water/TEA/ $\mathrm{MeOH}$ mixtures as well as sodium sulfide and L-ascorbic acid as scavengers (see Figure S-103). In all cases, CTF-15 and CTF-34 were the best-performing materials compared to CTF16 followed by CTF-3 Suzuki, which indicates strongly that superior photocatalytsts were obtained, rather than materials that were simply better optimized for the oxidation of TEA.

The five best-performing photocatalysts, CTF-15 (2,5-linked benzonitrile), CTF-34 (3,7-linked dibenzo[b,d]thiophene sulfone), CTF-30 (2,5-linked pyrazine), CTF-12 (2,5-linked selenophene), and CTF-19 (1,4-linked tetrafluorobenzene), were characterized by scanning electron microscopy (SEM; Figures S-25 and S-26), which shows that CTF-15 and CTF-16 consist of $20-50 \mu \mathrm{m}$ long and $1-2 \mu \mathrm{m}$ thick rods that are bundled together, while the other polymers show oval particles in the size range $8-23 \mu \mathrm{m}$ for CTF-34 and up to $50 \mu \mathrm{m}$ for CTF-12. Inductively coupled plasma optical emission spectrometry (ICP-OES) analysis was carried out for a subset and palladium levels between 0.44 for CTF-34 and 1.35 wt \% for CTF-5 were found.

Residual palladium has been shown to act as a co-catalyst for

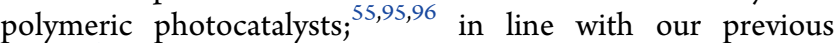
report, $^{57}$ we observe significant hydrogen production from CTF-15 and CTF-34 without added platinum (Figure S-96). However, we do not expect that this significantly affects the photocatalytic performance of these materials because platinum is added after the synthesis as the co-catalyst to saturate the performance. ${ }^{57}$

An external quantum efficiency (EQE) of $15.9 \%$ was determined with a light-emitting diode at $420 \mathrm{~nm}$ for CTF15 in water/TEA/MeOH with 3 wt \% Pt. This value is higher than that for the linear polymers poly ( $p$-phenylene) with an $\mathrm{EQE}_{420 \mathrm{~nm}}=0.4 \%^{38}$ and poly(dibenzo[b,d] thiophene sulfone) with an $\mathrm{EQE}_{420 \mathrm{~nm}}=11.6 \%{ }^{93}$ (both in water/TEA/MeOH), poly(benzothiadiazole-co-phenylene) with an $\mathrm{EQE}_{420 \mathrm{~nm}}=$ $4.0 \%{ }^{42}$ from water/TEOA/3 wt $\% \mathrm{Pt}$, but lower than optimized nanoparticles of poly(dibenzo $[b, d]$ thiophene sulfone $)\left(\mathrm{EQE}_{420 \mathrm{~nm}}=20.4 \%\right.$, water $\left./ \mathrm{TEA} / \mathrm{MeOH}\right),{ }^{97}$ co-polymer of dibenzo[ $[b, d]$ thiophene-dibenzo[ $[b, d]$ thiophene sulfone $\left(\mathrm{EQE}_{420 \mathrm{~nm}}=20.7 \%\right.$, water/TEA $\left./ \mathrm{MeOH}\right),{ }^{34}$ or optimized carbon nitrides $\left(\mathrm{EQE}_{405 \mathrm{~nm}}=50.7 \%, \mathrm{Pt} /\right.$ water $\left./ \mathrm{TEOA}\right) .^{98}$

The photostability of CTF-15 (2,5-linked benzonitrile CTF) was tested over $50 \mathrm{~h}$ under visible-light illumination (Figure 5,

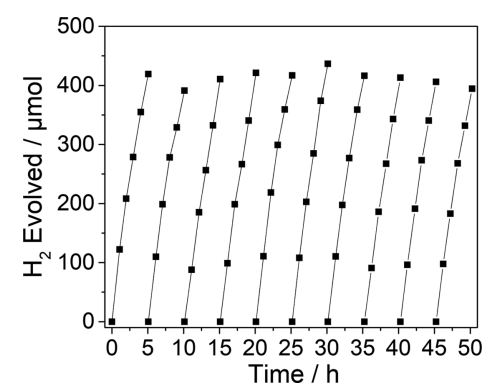

Figure 5. Extended photocatalysis run for CTF-15 (25 mg) in water/ $\mathrm{TEA} / \mathrm{MeOH}(1 / 1 / 1$ mixture) with $3 \mathrm{wt} \%$ platinum as co-catalyst under visible light $(\lambda>420 \mathrm{~nm}, 300 \mathrm{~W}$ Xe light source $)$.

$\lambda>420 \mathrm{~nm}$, Xe light source) and further $50 \mathrm{~h}$ on a solar simulator (Figure S-110). The CTF shows no change of activity or in post-illumination characterization, indicating good stability of this photocatalyst.

\section{CONCLUSIONS}

A library of 39 new CTFs was made via Suzuki-Miyaura polycondensation, where the modularity of this approach allowed us to obtain CTFs with a much richer structural diversity than has been studied previously. All materials were characterized and studied for their photocatalytic performance using a high-throughput workflow, as well as conventional kinetic measurements. The performance of the best-performing CTFs are among the highest for this material class. We showed that the photocatalytic performance of these CTFs can be analyzed by their predicted electron affinity, predicted ionization potential, optical gap, and dispersibility in the reaction mixture. Electron affinity and dispersibility were found to be the dominant variables under the conditions used.

In this study, we measured all materials using the rapid screening method (up to 50 samples per day) and traditional kinetic methods ( $1-2$ samples per day). This was done to establish the reliability of the screening method. The generally good correlation (Figure 4) between the screening results and the kinetic measurements suggests that this could be a powerful method in the future to discover new photocatalysts of any materials class, as well as providing valuable insights into the structure-property relationships across a diverse range of chemical functionality.

\section{ASSOCIATED CONTENT}

\section{S Supporting Information}

The Supporting Information is available free of charge on the ACS Publications website at DOI: 10.1021/acs.chemmater.9b02825. 
Synthetic procedures, FT-IR spectra, TGA traces, UVvis spectra, PXRD patterns, contact angle measurements, SEM images, PL spectra and TCSPC experiments, hydrogen evolution data, ICP-OES data, post-photocatalysis characterization data, and DFT results (PDF) Supporting structures, raw data, DFT calculations; predicted potentials and optical gap (ZIP)

\section{AUTHOR INFORMATION}

\section{Corresponding Authors}

*E-mail: m.zwijnenburg@ucl.ac.uk (M.A.Z.).

*E-mail: ssprick@liverpool.ac.uk (R.S.S.).

*E-mail: aicooper@liverpool.ac.uk (A.I.C.).

\section{ORCID}

Christian B. Meier: 0000-0003-4030-0350

Kim E. Jelfs: 0000-0001-7683-7630

Martijn A. Zwijnenburg: 0000-0001-5291-2130

Reiner Sebastian Sprick: 0000-0002-5389-2706

Andrew I. Cooper: 0000-0003-0201-1021

\section{Author Contributions}

All authors have given approval to the final version of the manuscript.

\section{Notes}

The authors declare no competing financial interest.

\section{ACKNOWLEDGMENTS}

We thank the Engineering and Physical Sciences Research Council (EPSRC) for financial support under Grants EP/ N004884/1 and EP/P005543/1. Dr. L. Wilbraham is acknowledged for useful discussion. K.E.J. thanks the Royal Society for a University Research Fellowship.

\section{REFERENCES}

(1) Savchenko, V. I.; Makaryan, I. A. Palladium Catalyst for the Production of Pure Margarine: Catalyst and New Non-filtration Technology Improve Production and Quality. Platinum Met. Rev. 1999, 43, 74-82.

(2) Elsherif, M.; Manan, Z. A.; Kamsah, M. Z. State-of-the-Art of Hydrogen Management in Refinery and Industrial Process Plants. J. Nat. Gas Sci. Eng. 2015, 24, 346-356.

(3) Häussinger, P.; Lohmüller, R.; Watson, A. M. Hydrogen, 6. Uses. Ullmann's Encyclopedia of Industrial Chemistry; Wiley-VCH Verlag GmbH \& Co. KGaA, 2000.

(4) Ramachandran, R.; Menon, R. K. An Overview of Industrial Uses of Hydrogen. Int. J. Hydrogen Energy 1998, 23, 593-598.

(5) Marbán, G.; Valdés-Solís, T. Towards the Hydrogen Economy? Int. J. Hydrogen Energy 2007, 32, 1625-1637.

(6) Barelli, L.; Bidini, G.; Gallorini, F.; Servili, S. Hydrogen Production through Sorption-Enhanced Steam Methane Reforming and Membrane Technology: A Review. Energy 2008, 33, 554-570.

(7) Fujishima, A.; Honda, K. Photolysis-Decomposition of Water at the Surface of an Irradiated Semiconductor. Nature 1972, 238, 3738.

(8) Chen, X.; Shen, S.; Guo, L.; Mao, S. S. Semiconductor-Based Photocatalytic Hydrogen Generation. Chem. Rev. 2010, 110, 65036570.

(9) Kudo, A.; Miseki, Y. Heterogeneous Photocatalyst Materials for Water Splitting. Chem. Soc. Rev. 2009, 38, 253-278.

(10) Yang, L.; Zhou, H.; Fan, T.; Zhang, D. Semiconductor Photocatalysts for Water Oxidation: Current Status and Challenges. Phys. Chem. Chem. Phys. 2014, 16, 6810-6826.

(11) Yanagida, S.; Kabumoto, A.; Mizumoto, K.; Pac, C.; Yoshino, K. Poly(p-phenylene)-Catalysed Photoreduction of Water to Hydrogen. J. Chem. Soc., Chem. Commun. 1985, 0, 474-475.
(12) Shibata, T.; Kabumoto, A.; Shiragami, T.; Ishitani, O.; Pac, C.; Yanagida, S. Novel visible-light-driven photocatalyst. Poly( $p$-phenylene)-Catalyzed Photoreductions of Water, Carbonyl Compounds, and Olefins. J. Phys. Chem. A. 1990, 94, 2068-2076.

(13) Matsuoka, S.; Kohzuki, T.; Pac, C.; Ishida, A.; Takamuku, S.; Kusaba, M.; Nakashima, N.; Yanagida, S. Photocatalysis of Oligo( $p$ Phenylenes). Photochemical Reduction of Carbon Dioxide with Triethylamine. J. Phys. Chem. A. 1992, 96, 4437-4442.

(14) Matsuoka, S.; Kohzuki, T.; Kuwana, Y.; Nakamura, A.; Yanagida, S. Visible-Light-Induced Photocatalysis of Poly(Pyridine2,5-Diyl). Photoreduction of Water, Carbonyl Compounds and Alkenes with Triethylamine. J. Chem. Soc., Perkin Trans. 2 1992, 679-685.

(15) Wang, X.; Maeda, K.; Thomas, A.; Takanabe, K.; Xin, G.; Carlsson, J. M.; Domen, K.; Antonietti, M. A Metal-Free Polymeric Photocatalyst for Hydrogen Production from Water under Visible Light. Nat. Mater. 2009, 8, 76-80.

(16) Schwinghammer, K.; Tuffy, B.; Mesch, M. B.; Wirnhier, E.; Martineau, C.; Taulelle, F.; Schnick, W.; Senker, J.; Lotsch, B. V. Triazine-Based Carbon Nitrides for Visible-Light-Driven Hydrogen Evolution. Angew. Chem., Int. Ed. 2013, 52, 2435-2439.

(17) Lau, V. W. H.; Mesch, M. B.; Duppel, V.; Blum, V.; Senker, J.; Lotsch, B. V. Low-Molecular-Weight Carbon Nitrides for Solar Hydrogen Evolution. J. Am. Chem. Soc. 2015, 137, 1064-1072.

(18) Liu, J.; Wang, H.; Antonietti, M. Graphitic Carbon Nitride "Reloaded": Emerging Applications beyond (Photo)Catalysis. Chem. Soc. Rev. 2016, 45, 2308-2326.

(19) Wang, Y.; Wang, X.; Antonietti, M. Polymeric Graphitic Carbon Nitride as a Heterogeneous Organocatalyst: From Photochemistry to Multipurpose Catalysis to Sustainable Chemistry. Angew. Chem., Int. Ed. 2012, 51, 68-89.

(20) Schwinghammer, K.; Hug, S.; Mesch, M. B.; Senker, J.; Lotsch, B. V. Phenyl-Triazine Oligomers for Light-Driven Hydrogen Evolution. Energy Environ. Sci. 2015, 8, 3345-3353.

(21) Chuang, P.-K.; Wu, K.-H.; Yeh, T.-F.; Teng, H. Extending the $\pi$-Conjugation of g-C3N4 by Incorporating Aromatic Carbon for Photocatalytic H2 Evolution from Aqueous Solution. ACS Sustainable Chem. Eng. 2016, 4, 5989-5997.

(22) Zhang, J.; Sun, J.; Maeda, K.; Domen, K.; Liu, P.; Antonietti, M.; Fu, X.; Wang, X. Sulfur-Mediated Synthesis of Carbon Nitride: Band-Gap Engineering and Improved Functions for Photocatalysis. Energy Environ. Sci. 2011, 4, 675.

(23) Hu, S.; Li, F.; Fan, Z.; Wang, F.; Zhao, Y.; Lv, Z. Band GapTunable Potassium Doped Graphitic Carbon Nitride with Enhanced Mineralization Ability. Dalton Trans. 2015, 44, 1084-1092.

(24) Li, Y.; Jin, R.; Li, G.; Liu, X.; Yu, M.; Xing, Y.; Shi, Z. Preparation of Phenyl Group Functionalized G-C3N4 Nanosheets with Extended Electron Delocalization for Enhanced Visible-Light Photocatalytic Activity. New J. Chem. 2018, 42, 6756-6762.

(25) Zhang, J.; Zhang, G.; Chen, X.; Lin, S.; Möhlmann, L.; Dołęga, G.; Lipner, G.; Antonietti, M.; Blechert, S.; Wang, X. Co-Monomer Control of Carbon Nitride Semiconductors to Optimize Hydrogen Evolution with Visible Light. Angew. Chem., Int. Ed. 2012, 51, 31833187.

(26) Kailasam, K.; Schmidt, J.; Bildirir, H.; Zhang, G.; Blechert, S.; Wang, X.; Thomas, A. Room Temperature Synthesis of HeptazineBased Microporous Polymer Networks as Photocatalysts for Hydrogen Evolution. Macromol. Rapid Commun. 2013, 34, 1008-1013.

(27) Fu, J.; Yu, J.; Jiang, C.; Cheng, B. G- $\mathrm{C}_{3} \mathrm{~N}_{4}$-Based Heterostructured Photocatalysts. Adv. Energy Mater. 2017, 8, No. 1701503

(28) Fan, X.; Zhang, L.; Cheng, R.; Wang, M.; Li, M.; Zhou, Y.; Shi, J. Construction of Graphitic $\mathrm{C}_{3} \mathrm{~N}_{4}$-Based Intramolecular DonorAcceptor Conjugated Copolymers for Photocatalytic Hydrogen Evolution. ACS Catal. 2015, 5, 5008-5015.

(29) Leclerc, M., Morin, J.-F., Eds. Design and Synthesis of Conjugated Polymers; Wiley-VCH Verlag GmbH \& Co. KGaA: Weinheim, Germany, 2010. 
(30) Wang, K.; Yang, L.-M.; Wang, X.; Guo, L.; Cheng, G.; Zhang, C.; Jin, S.; Tan, B.; Cooper, A. Covalent Triazine Frameworks via a Low-Temperature Polycondensation Approach. Angew. Chem., Int. Ed. 2017, 56, 14149-14153.

(31) Zhang, G.; Lan, Z.-A.; Wang, X. Conjugated Polymers: Catalysts for Photocatalytic Hydrogen Evolution. Angew. Chem., Int. Ed. 2016, 55, 15712-15727.

(32) Xu, Y.; Jin, S.; Xu, H.; Nagai, A.; Jiang, D. Conjugated Microporous Polymers: Design, Synthesis and Application. Chem. Soc. Rev. 2013, 42, 8012.

(33) Sprick, R. S.; Aitchison, C. M.; Berardo, E.; Turcani, L.; Wilbraham, L.; Alston, B. M.; Jelfs, K. E.; Zwijnenburg, M. A.; Cooper, A. I. Maximising the Hydrogen Evolution Activity in Organic Photocatalysts by Co-Polymerisation. J. Mater. Chem. A 2018, 6, 11994-12003.

(34) Bai, Y.; Wilbraham, L.; Slater, B. J.; Zwijnenburg, M. A.; Sprick, R. S.; Cooper, A. I. Accelerated Discovery of Organic Polymer Photocatalysts for Hydrogen Evolution from Water through the Integration of Experiment and Theory. J. Am. Chem. Soc. 2019, 141, 9063-9071.

(35) Matsuoka, S.; Fujii, H.; Yamada, T.; Pac, C.; Ishida, A.; Takamuku, S.; Kusaba, M.; Nakashima, N.; Yanagida, S.; Hashimoto, K.; et al. Photocatalysis of Oligo(para-phenylenes) - Photoreductive Production of Hydrogen and Ethanol in Aqueous Triethylamine. J. Phys. Chem. A. 1991, 95, 5802-5808.

(36) Matsuoka, S.; Fujii, H.; Pac, C.; Yanagida, S. Photocatalysis of Oligo( $p$-Phenylene) Leading to Reductive Formation of Hydrogen and Ethanol from Triethylamine in Aqueous Organic Solvent. Chem. Lett. 1990, 19, 1501-1502.

(37) Maruo, K.; Wada, Y.; Yanagida, S. Photocatalysis of Perfluorinated Oligo( $p$-Phenylene)s. Bull. Chem. Soc. Jpn. 1992, 65, 3439-3449.

(38) Sprick, R. S.; Bonillo, B.; Clowes, R.; Guiglion, P.; Brownbill, N. J.; Slater, B. J.; Blanc, F.; Zwijnenburg, M. A.; Adams, D. J.; Cooper, A. I. Visible-Light-Driven Hydrogen Evolution Using Planarized Conjugated Polymer Photocatalysts. Angew. Chem., Int. Ed. 2016, 55, $1792-1796$.

(39) Woods, D. J.; Sprick, R. S.; Smith, C. L.; Cowan, A. J.; Cooper, A. I. A Solution-Processable Polymer Photocatalyst for Hydrogen Evolution from Water. Adv. Energy Mater. 2017, 7, No. 1700479.

(40) Maruo, K.; Yamada, K.; Wada, Y.; Yanagida, S. Visible-Light Induced Photocatalysis of Partially Fluorinated Poly( $p$-Phenylene) and Related Linear Phenylene Derivatives. Bull. Chem. Soc. Jpn. 1993, 66, 1053-1064.

(41) Yanagida, S.; Ogata, T.; Kuwana, Y.; Wada, Y.; Murakoshi, K.; Ishida, A.; Takamuku, S.; Kusaba, M.; Nakashima, N. Synthesis of $2,{ }^{\prime}: 5^{\prime}, 2^{\prime \prime}$-Terpyridine and $2,2^{\prime}: 5^{\prime}, 2^{\prime \prime}: 5^{\prime \prime}, 2^{\prime \prime \prime}$-Quaterpyridine and their Photocatalysis of the Reduction of Water. J. Chem. Soc., Perkin Trans. 2 1996, 1963-1969.

(42) Yang, C.; Ma, B. C.; Zhang, L.; Lin, S.; Ghasimi, S.; Landfester, K.; Zhang, K. A. I.; Wang, X. Molecular Engineering of Conjugated Polybenzothiadiazoles for Enhanced Hydrogen Production by Photosynthesis. Angew. Chem., Int. Ed. 2016, 55, 9202-9206.

(43) Sprick, R. S.; Wilbraham, L.; Bai, Y.; Guiglion, P.; Monti, A.; Clowes, R.; Cooper, A. I.; Zwijnenburg, M. A. Nitrogen Containing Linear Poly(Phenylene) Derivatives for Photo-Catalytic Hydrogen Evolution from Water. Chem. Mater. 2018, 30, 5733-5742.

(44) Dai, C.; Xu, S.; Liu, W.; Gong, X.; Panahandeh-Fard, M.; Liu, Z.; Zhang, D.; Xue, C.; Loh, K. P.; Liu, B. Dibenzothiophene- S,SDioxide-Based Conjugated Polymers: Highly Efficient Photocatalyts for Hydrogen Production from Water under Visible Light. Small 2018, No. 1801839.

(45) Lan, Z.-A.; Zhang, G.; Chen, X.; Zhang, Y.; Zhang, K. A. I.; Wang, X. Reducing the Exciton Binding Energy of Donor-AcceptorBased Conjugated Polymers to Promote Charge-Induced Reactions. Angew. Chem., Int. Ed. 2019, 58, 10236-10240.

(46) Vyas, V. S.; Haase, F.; Stegbauer, L.; Savasci, G.; Podjaski, F.; Ochsenfeld, C.; Lotsch, B. V. A Tunable Azine Covalent Organic
Framework Platform for Visible Light-Induced Hydrogen Generation. Nat. Commun. 2015, 6, No. 8508.

(47) Stegbauer, L.; Schwinghammer, K.; Lotsch, B. V. A HydrazoneBased Covalent Organic Framework for Photocatalytic Hydrogen Production. Chem. Sci. 2014, 5, 2789-2793.

(48) Haase, F.; Banerjee, T.; Savasci, G.; Ochsenfeld, C.; Lotsch, B. V. Structure-property-activity Relationships in a Pyridine Containing Azine-Linked Covalent Organic Framework for Photocatalytic Hydrogen Evolution $\dagger$. Faraday Discuss. 2017, 201, 247-264.

(49) Wang, X.; Chen, L.; Chong, S. Y.; Little, M. A.; Wu, Y.; Zhu, W.-H.; Clowes, R.; Yan, Y.; Zwijnenburg, M. A.; Sprick, R. S.; et al. Sulfone-Containing Covalent Organic Frameworks for Photocatalytic Hydrogen Evolution from Water. Nat. Chem. 2018, 10, 1180-1189.

(50) Stegbauer, L.; Zech, S.; Savasci, G.; Banerjee, T.; Podjaski, F.; Schwinghammer, K.; Ochsenfeld, C.; Lotsch, B. V. Tailor-Made Photoconductive Pyrene-Based Covalent Organic Frameworks for Visible-Light Driven Hydrogen Generation. Adv. Energy Mater. 2018, 8, No. 1703278.

(51) Banerjee, T.; Gottschling, K.; Savasci, G.; Ochsenfeld, C.; Lotsch, B. V. $\mathrm{H}_{2}$ Evolution with Covalent Organic Framework Photocatalysts. ACS Energy Lett. 2018, 3, 400-409.

(52) Zhou, J.; Lei, Y.; Ma, C.; Lv, W.; Li, N.; Wang, Y.; Xu, H.; Zou, Z. A (001) Dominated Conjugated Polymer with High-Performance of Hydrogen Evolution under Solar Light Irradiation. Chem. Commun. 2017, 53, 10536-10539.

(53) Pachfule, P.; Acharjya, A.; Roeser, J.; Langenhahn, T.; Schwarze, M.; Schomäcker, R.; Thomas, A.; Schmidt, J. Diacetylene Functionalized Covalent Organic Framework (COF) for Photocatalytic Hydrogen Generation. J. Am. Chem. Soc. 2018, 140, 14231427.

(54) Banerjee, T.; Haase, F.; Savasci, G.; Gottschling, K.; Ochsenfeld, C.; Lotsch, B. V. Single-Site Photocatalytic H2 Evolution from Covalent Organic Frameworks with Molecular Cobaloxime CoCatalysts. J. Am. Chem. Soc. 2017, 139, 16228-16234.

(55) Li, L.; Cai, Z.; Wu, Q.; Lo, W. Y.; Zhang, N.; Chen, L. X.; Yu, L. Rational Design of Porous Conjugated Polymers and Roles of Residual Palladium for Photocatalytic Hydrogen Production. J. Am. Chem. Soc. 2016, 138, 7681-7686.

(56) Li, L.; Lo, W. Y.; Cai, Z.; Zhang, N.; Yu, L. Donor-Acceptor Porous Conjugated Polymers for Photocatalytic Hydrogen Production: The Importance of Acceptor Comonomer. Macromolecules 2016, 49, 6903-6909.

(57) Meier, C. B.; Sprick, R. S.; Monti, A.; Guiglion, P.; Lee, J.-S. M.; Zwijnenburg, M. A.; Cooper, A. I. Structure-Property Relationships for Covalent Triazine-Based Frameworks: The Effect of Spacer Length on Photocatalytic Hydrogen Evolution from Water. Polymer 2017, 126, 283-290.

(58) Sprick, R. S.; Bonillo, B.; Sachs, M.; Clowes, R.; Durrant, J. R.; Adams, D. J.; Cooper, A. I. Extended Conjugated Microporous Polymers for Photocatalytic Hydrogen Evolution from Water. Chem. Commun. 2016, 52, 10008-10011.

(59) Bi, S.; Lan, Z.; Paasch, S.; Zhang, W.; He, Y.; Zhang, C.; Liu, F.; Wu, D.; Zhuang, X.; Brunner, E.; et al. Substantial Cyano-Substituted Fully $\mathrm{Sp}^{2}$-Carbon-Linked Framework: Metal-Free Approach and Visible-Light-Driven Hydrogen Evolution. Adv. Funct. Mater. 2017, 27, No. 1703146.

(60) Guo, L.; Niu, Y.; Xu, H.; Li, Q.; Razzaque, S.; Huang, Q.; Jin, S.; Tan, B. Engineering Heteroatoms with Atomic Precision in Donor-acceptor Covalent Triazine Frameworks to Boost Photocatalytic Hydrogen Production. J. Mater. Chem. A 2018, 6, 1977519781.

(61) Zhao, Y.; Ma, W.; Xu, Y.; Zhang, C.; Wang, Q.; Yang, T.; Gao, X.; Wang, F.; Yan, C.; Jiang, J.-X. Effect of Linking Pattern of Dibenzothiophene-S,S-Dioxide-Containing Conjugated Microporous Polymers on the Photocatalytic Performance. Macromolecules 2018, 51, 9502-9508.

(62) Sprick, R. S.; Jiang, J. X.; Bonillo, B.; Ren, S.; Ratvijitvech, T.; Guiglion, P.; Zwijnenburg, M. A.; Adams, D. J.; Cooper, A. I. Tunable 
Organic Photocatalysts for Visible-Light-Driven Hydrogen Evolution. J. Am. Chem. Soc. 2015, 137, 3265-3270.

(63) Chu, S.; Wang, Y.; Guo, Y.; Zhou, P.; Yu, H.; Luo, L.; Kong, F.; Zou, Z. Facile Green Synthesis of Crystalline Polyimide Photocatalyst for Hydrogen Generation from Water. J. Mater. Chem. 2012, 22, $15519-15521$.

(64) Zhang, Z.; Long, J.; Yang, L.; Chen, W.; Dai, W.; Fu, X.; Wang, X. Organic Semiconductor for Artificial Photosynthesis: Water Splitting into Hydrogen by a Bioinspired $\mathrm{C}_{3} \mathrm{~N}_{3} \mathrm{~S}_{3}$ polymer under Visible Light Irradiation. Chem. Sci. 2011, 2, 1826-1830.

(65) Xie, J.; Shevlin, S. A.; Ruan, Q.; Moniz, S. J. A.; Liu, Y.; Liu, X.; Li, Y.; Lau, C. C.; Guo, Z. X.; Tang, J. Efficient Visible Light-Driven Water Oxidation and Proton Reduction by an Ordered Covalent Triazine-Based Framework. Energy Environ. Sci. 2018, 11, 1617-1624.

(66) Lan, Z.-A.; Fang, Y.; Zhang, Y.; Wang, X. Photocatalytic Oxygen Evolution from Functional Triazine-Based Polymers with Tunable Band Structure. Angew. Chem., Int. Ed. 2018, 57, 470-474.

(67) Bi, J.; Fang, W.; Li, L.; Wang, J.; Liang, S.; He, Y.; Liu, M.; Wu, L. Covalent Triazine-Based Frameworks as Visible Light Photocatalysts for the Splitting of Water. Macromol. Rapid Commun. 2015, $36,1799-1805$.

(68) Huang, W.; Wang, Z. J.; Ma, B. C.; Ghasimi, S.; Gehrig, D.; Laquai, E.; Landfester, K.; Zhang, K. A. I. Hollow Nanoporous Covalent Triazine Frameworks via Acid Vapor-Assisted Solid Phase Synthesis for Enhanced Visible Light Photoactivity. J. Mater. Chem. A 2016, 4, 7555-7559.

(69) Li, L.; Fang, W.; Zhang, P.; Bi, J.; He, Y.; Wang, J.; Su, W. Sulfur-Doped Covalent Triazine-Based Frameworks for Enhanced Photocatalytic Hydrogen Evolution from Water under Visible Light. J. Mater. Chem. A 2016, 4, 12402-12406.

(70) Cheng, Z.; Fang, W.; Zhao, T.; Fang, S.; Bi, J.; Liang, S.; Li, L.; Yu, Y.; Wu, L. Efficient Visible-Light-Driven Photocatalytic Hydrogen Evolution on Phosphorus-Doped Covalent Triazine-Based Frameworks. ACS Appl. Mater. Interfaces 2018, 10, 41415-41421.

(71) Kuhn, P.; Antonietti, M.; Thomas, A. Porous, Covalent Triazine-Based Frameworks Prepared by Ionothermal Synthesis. Angew. Chem., Int. Ed. 2008, 47, 3450-3453.

(72) Bojdys, M. J.; Jeromenok, J.; Thomas, A.; Antonietti, M. Rational Extension of the Family of Layered, Covalent, TriazineBased Frameworks with Regular Porosity. Adv. Mater. 2010, 22, 2202-2205.

(73) Bhunia, A.; Esquivel, D.; Dey, S.; Fernández-Terán, R. J.; Goto, Y.; Inagaki, S.; Van Der Voort, P.; Janiak, C. A Photoluminescent Covalent Triazine Framework: $\mathrm{CO}_{2}$ Adsorption, Light-Driven Hydrogen Evolution and Sensing of Nitroaromatics. J. Mater. Chem. A 2016, 4, 13450-13457.

(74) Kuecken, S.; Acharjya, A.; Zhi, L.; Schwarze, M.; Schomacker, R.; Thomas, A. Fast Tuning of Covalent Triazine Frameworks for Photocatalytic Hydrogen Evolution. Chem. Commun. 2017, 53, 58545857.

(75) Lan, Z.-A.; Fang, Y.; Chen, X.; Wang, X. Thermal AnnealingInduced Structural Reorganization in Polymeric Photocatalysts for Enhanced Hydrogen Evolution. Chem. Commun. 2019, 55, 77567759.

(76) Cheng, Z.; Zheng, K.; Lin, G.; Fang, S.; Li, L.; Bi, J.; Shen, J.; $\mathrm{Wu}, \mathrm{L}$. Constructing a Novel Family of Halogen Doped Covalent Triazine-Based Frameworks as Efficient Metal-Free Photocatalysts for Hydrogen Production. Nanoscale Adv. 2019, 1, 2674-2680.

(77) Schwarz, D.; Acharja, A.; Ichangi, A.; Lyu, P.; Opanasenko, M. V.; Goßler, F. R.; König, T. A. F.; Čejka, J.; Nachtigall, P.; Thomas, A.; et al. Fluorescent Sulphur- and Nitrogen-Containing Porous Polymers with Tuneable Donor-Acceptor Domains for Light-Driven Hydrogen Evolution. Chem.-Eur. J. 2018, 24, 11916-11921.

(78) Kuhn, P.; Thomas, A.; Antonietti, M. Toward Tailorable Porous Organic Polymer Networks: A High-Temperature Dynamic Polymerization Scheme Based on Aromatic Nitriles. Macromolecules 2009, 42, 319-326.

(79) Li, L.; Fang, W.; Zhang, P.; Bi, J.; He, Y.; Wang, J.-Y.; Su, W. Sulfur-Doped Covalent Triazine-Based Frameworks for Enhanced
Photocatalytic Hydrogen Evolution from Water under Visible Light. J. Mater. Chem. A 2016, 4, 12402-12406.

(80) Huang, W.; He, Q.; Hu, Y.; Li, Y. Molecular Heterostructures of Covalent Triazine Frameworks for Enhanced Photocatalytic Hydrogen Production. Angew. Chem. 2019, 131, 8768-8772.

(81) Yu, K.; Bi, S.; Ming, W.; Wei, W.; Zhang, Y.; Xu, J.; Qiang, P.; Qiu, F.; Wu, D.; Zhang, F. Side-Chain-Tuned $\pi$-Extended Porous Polymers for Visible Light-Activated Hydrogen Evolution. Polym. Chem. 2019, 10, 3758-3763.

(82) Turcani, L.; Berardo, E.; Jelfs, K. E. STK: A Python Toolkit for Supramolecular Assembly. J. Comput. Chem. 2018, 1931-1942.

(83) Turcani, L. GitHub-supramolecular-toolkit/stk. https:// github.com/supramolecular-toolkit/stk (accessed Sept 1, 2018).

(84) Landrum, G. RDKit: Open-Source Cheminformatics. https:// www.rdkit.org (accessed Sept 1, 2018).

(85) Jorgensen, W. L.; Maxwell, D. S.; Tirado-Rives, J. Development and Testing of the OPLS All-Atom Force Field on Conformational Energetics and Properties of Organic Liquids. J. Am. Chem. Soc. 1996, $118,11225-11236$

(86) Banks, J. L.; Beard, H. S.; Cao, Y.; Cho, A. E.; Damm, W.; Farid, R.; Felts, A. K.; Halgren, T. A.; Mainz, D. T.; Maple, J. R.; et al. Integrated Modeling Program, Applied Chemical Theory (IMPACT). J. Comput. Chem. 2005, 26, 1752-1780.

(87) Becke, A. D. Density-functional Thermochemistry. III. The Role of Exact Exchange. J. Chem. Phys. 1993, 98, 5648-5652.

(88) Stephens, P. J.; Devlin, F. J.; Chabalowski, C. F.; Frisch, M. J. $\mathrm{Ab}$ Initio Calculation of Vibrational Absorption and Circular Dichroism Spectra Using Density Functional Force Fields. J. Phys. Chem. A. 1994, 98, 11623-11627.

(89) Schäfer, A.; Horn, H.; Ahlrichs, R. Fully Optimized Contracted Gaussian Basis Sets for Atoms Li to Kr. J. Chem. Phys. 1992, 97, 2571-2577.

(90) Guiglion, P.; Butchosa, C.; Zwijnenburg, M. A. Polymeric Watersplitting Photocatalysts; A Computational Perspective on the Water Oxidation Conundrum. J. Mater. Chem. A 2014, 2, 1199612004

(91) Guiglion, P.; Monti, A.; Zwijnenburg, M. A. Validating a Density Functional Theory Approach for Predicting the Redox Potentials Associated with Charge Carriers and Excitons in Polymeric Photocatalysts. J. Phys. Chem. C 2017, 121, 1498-1506.

(92) Klamt, A.; Schüürmann, G. COSMO: A New Approach to Dielectric Screening in Solvents with Explicit Expressions for the Screening Energy and Its Gradient. J. Chem. Soc., Perkin Trans. 2 1993, 5, 799-805.

(93) Sachs, M.; Sprick, R. S.; Pearce, D.; Hillman, S. A. J.; Monti, A.; Guilbert, A. A. Y.; Brownbill, N. J.; Dimitrov, S.; Shi, X.; Blanc, F.; et al. Understanding Structure-Activity Relationships in Linear Polymer Photocatalysts for Hydrogen Evolution. Nat. Commun. 2018, 9, No. 4968.

(94) Schwarze, M.; Stellmach, D.; Schröder, M.; Kailasam, K.; Reske, R.; Thomas, A.; Schomäcker, R. Quantification of Photocatalytic Hydrogen Evolution. Phys. Chem. Chem. Phys. 2013, 15, 3466.

(95) Kosco, J.; Sachs, M.; Godin, R.; Kirkus, M.; Francas, L.; Bidwell, M.; Qureshi, M.; Anjum, D.; Durrant, J. R.; McCulloch, I. The Effect of Residual Palladium Catalyst Contamination on the Photocatalytic Hydrogen Evolution Activity of Conjugated Polymers. Adv. Energy Mater. 2018, 8, No. 1802181.

(96) Sprick, R. S.; Bai, Y.; Guilbert, A. A. Y.; Zbiri, M.; Aitchison, C. M.; Wilbraham, L.; Yan, Y.; Woods, D. J.; Zwijnenburg, M. A.; Cooper, A. I. Photocatalytic Hydrogen Evolution from Water Using Fluorene and Dibenzothiophene Sulfone-Conjugated Microporous and Linear Polymers. Chem. Mater. 2019, 31, 305-313.

(97) Aitchison, C. M.; Sprick, R. S.; Cooper, A. I. Emulsion Polymerization Derived Organic Photocatalysts for Improved LightDriven Hydrogen Evolution. J. Mater. Chem. A 2019, 7, 2490-2496. (98) Lin, L.; Ou, H.; Zhang, Y.; Wang, X. Tri-s-Triazine-Based Crystalline Graphitic Carbon Nitrides for Highly Efficient Hydrogen Evolution Photocatalysis. ACS Catal. 2016, 6, 3921-3931. 